\title{
Essais
}

Revue interdisciplinaire d'Humanités

\section{Erreurs photographiques et création artistique dans les œuvres de Douglas Huebler et de John Baldessari}

François Maheu

\section{(2) OpenEdition \\ Journals \\ Édition électronique \\ URL : http://journals.openedition.org/essais/5358 \\ DOI : 10.4000/essais.5358 \\ ISSN : 2276-0970 \\ Éditeur \\ École doctorale Montaigne Humanités}

\section{Édition imprimée}

Date de publication : 15 mars 2016

Pagination : 158-173

ISBN : 978-2-9544269-7-6

ISSN : 2417-4211

Référence électronique

François Maheu, « Erreurs photographiques et création artistique dans les œuvres de Douglas Huebler et de John Baldessari », Essais [En ligne], 8 | 2016, mis en ligne le 28 octobre 2020, consulté le 01 novembre 2020. URL : http://journals.openedition.org/essais/5358 ; DOI : https://doi.org/10.4000/ essais.5358 


\section{Erreurs photographiques et création artistique dans les œuvres de Douglas Huebler et de John Baldessari}

\section{François Maheu}

L'art conceptuel offre un large éventail d'œuvres résultant de stratégies incluant l'erreur. L'étude de l'erreur dans certaines de ces œuvres nous invite à réévaluer la place du médium photographique dans ce courant artistique en particulier mais également au sein de l'art contemporain. L'erreur photographique incluse dans les œuvres envisagées constitue un défi lancé au spectateur, une sorte d'énigme que celui-ci aura à décoder. Ces erreurs sont le fait d'artistes conscients que «l'observation des violations de la norme doit fournir des indications sur le système et son fonctionnement. " ${ }^{1}$ Il faut noter qu'à la violation de la norme, les artistes conceptuels ajoutent également la transgression de la fonction. La norme et la fonction constituent deux paramètres à partir desquels il est permis de juger la déficience d'une photographie.

L'enjeu principal de cet article est d'aborder les démarches de deux artistes incontournables de l'art conceptuel à travers trois œuvres photographiques incluant une forme d'erreur. L'étude de chaque ouvre nous permettra de comprendre, non seulement les intentions des artistes, mais également les mécanismes de mise en œuvre de l'erreur photographique ainsi que leur portée théorique. Dans un premier temps, Douglas Huebler (1924-1997) nous initie, dans la Duration Piece \#4, Bradford, 09/1968, à une recherche épistémologique. Afin de bien comprendre sa démarche, nous aurons notamment recours à certaines notions de linguistique. Nous verrons également se dessiner des liens entre le travail de l'artiste et la théorie du Nouveau Roman. Dans un second temps, nous nous pencherons sur le travail de John Baldessari (né en 1931). À travers Wrong (1966-1968), l'artiste mène une critique de la pédagogie artistique. Nous ferons appel à une notion de base de la psychanalyse pour tenter de comprendre ce qui pousse cet artiste à exposer une erreur. Dans la troisième ouvre, Throwing Three Balls In The Air... (1973), l'artiste californien nous livre, grâce à une mise à contribution du hasard en vue de la production de l'échec, un commentaire sur la démarche de création artistique.

1 Georges Mounin (éd.), Dictionnaire de la linguistique, Paris, PUF, 1974, p. 157. 
Ces deux artistes sont issus de milieux culturels relativement différents. Douglas Huebler est familier de la scène newyorkaise tandis que John Baldessari est baigné dans l'atmosphère de la côte ouest. Tous deux ont d'abord développé une pratique picturale avant d'utiliser la photographie au sein de leurs œuvres. Un autre dénominateur commun aux deux artistes est leur expérience de pédagogue au California Institute of Art (CalArts) de Valencia ${ }^{2}$. Les œuvres que nous envisageons ont été produites entre 1966 et 1973.

\section{La performativité de l'erreur photographique}

En 1996, dans son article "Devons-nous tuer papa? " publié dans le catalogue de l'exposition "Origine and destination ", Mike Kelley attire notre attention sur le travail de Douglas Huebler. Il écrit : « L'œuvre de Doug n’est pas conçue pour échouer. ${ }^{4}$ Et pourtant, des échecs, des erreurs, des ratés, on en croise beaucoup dans les "location, duration et variable pieces " ${ }^{5}$ de Douglas Huebler. Comment peut-on, sinon concilier, au moins rapprocher ces deu constats ? Cette question constitue le nœud de l'étude de cette œuvre réalisée en 1968. À l'instar des autres œuvres de l'artiste, la Location Piece \#4, Bradford, 1968, est composée d'un protocole dactylographié et d'une série de photographies.

Prenons l'étude du protocole comme point de départ de notre analyse.

La Duration Piece \# 4, Bradford, 09/1968 illustre une citation tirée d'une interview que l'artiste accorda à Patricia Norvell le 25 juillet 1969 :

Trying to show that the system, or the idea, the thing that you've set up as the structure within which you will work, is what the art's about. ${ }^{6}$

L'analyse de cette ouvre nous permet de mieux comprendre comment Douglas Huebler, tout en limitant le potentiel narratif de la série photographique, c'est-à-dire sa capacité propre à construire et véhiculer une histoire, attire l'attention du spectateur vers la structure de l'œuvre, laquelle constitue le véritable enjeu de sa démarche.

2 Douglas Huebler y enseigna de 1976 à 1988 et John Baldessari de 1969 à 1986.

3 Anne Pontégnie et Marianne Van Leeuw (éds.), Origin and destination: Alighiero e Boetti, Douglas Huebler, Bruxelles, Société des Expositions du Palais des Beaux-Arts de Bruxelles, 1997, p. 155-173.

4 Anne Pontégnie et Marianne Van Leeuw (éds.), Origin and destination: Alighiero e Boetti, Douglas Huebler, Bruxelles, Société des Expositions du Palais des Beaux-Arts de Bruxelles, 1997, p. 171.

5 Titre générique donné à ses œuvres. La numérotation des œuvres recommence théoriquement à \#1 chaque année. Les premières œuvres d'Huebler apparaissent sur la scène artistique en 1966. Certaines œuvres étaient encore en cours de réalisation à sa mort.

6 "L'art consiste à essayer de montrer le système, ou l'idée, la chose que vous avez mise en place et à l'intérieur de laquelle vous allez travailler. ", Notre traduction. Alexander Alberro et Patricia Norvell, Recording Conceptual Art, Berkley, University of California Press, 2001, p. 148. 


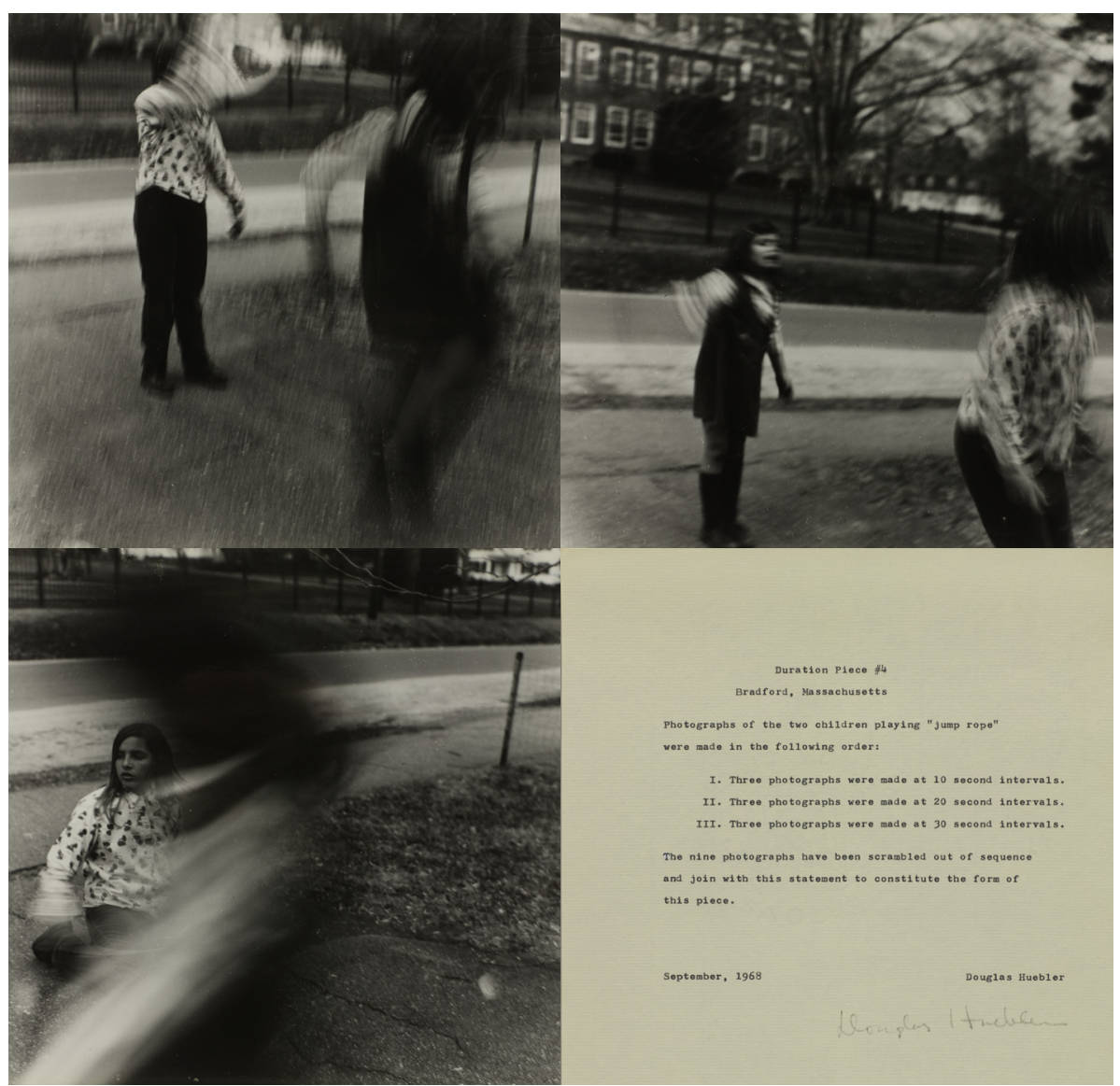

Duration Piece \#4

Bradford, Massachusetts

Des photos de deux enfants sautant à la corde ont été prises dans l'ordre suivant :

I. Trois photos ont été prises à 10 secondes d'intervalle.

II. Trois photos ont été prises à 20 secondes d'intervalle.

III. Trois photos ont été prises à 30 secondes d'intervalle.

Les 9 photos ont été mélangées, elles se joignent à la présente déclaration pour constituer la forme de cette œuvre.

Septembre, $1968 .^{7}$

Douglas Huebler, Duration Piece \#4, Bradford, Massachusetts, September 1968, 9 photographies et texte (détails). Courtesy Paula Cooper Gallery, New York. (C) SABAM, Belgium, 2015.

7 Traduction du protocole fournie par Frédéric Paul, in Frédéric Paul, Douglas Huebler, "Variable », etc., FRAC en Limousin, 1992, p. 51. 
L’œuvre fut réalisée en 1968 à Bradford dans le Massachusetts. Elle est composée de neuf photographies de format carré ainsi que d'un texte dactylographié reprenant le protocole de l'œuvre. Étymologiquement, le protocole

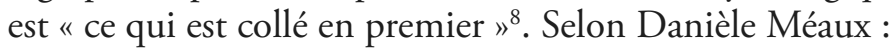

lorsqu'un protocole se trouve conjugué à la pratique de la photographie, il désigne ce qui se trouve posé en amont du "faire »- que ce «faire " concerne la réalisation des images ou leur interprétation. Ainsi, le protocole est constitué des règles précises qui régissent et déterminent l'exercice de la prise de vue ; mais, une de ses particularités est d'être affiché comme tel, sa nature guide aussi l'observation et la compréhension du travail par le récepteur.'

L'intégration du protocole dans l'œuvre photographique conceptuelle de Douglas Huebler, tout en guidant l'observation et l'interprétation du spectateur, crée un champ spécifique dans lequel se pose la question du respect ou du non-respect, lors de la prise de vue, des conditions et des règles que l'artiste s'est lui-même imposées.

Chacune des neuf photographies représente deux fillettes sautant à la corde le long d'une rue de banlieue. Chaque photographie comporte un flou de mouvement lié à une vitesse d'obturation trop basse pour l'enregistrement du jeu en question. Dans ce cas de figure, l'erreur photographique procède de la forme de l'œuvre. L'imprécision liée au flou ne permet pas de remplir pleinement la fonction d'enregistrement de la photographie. Par ailleurs, ce type de flou ne renvoie pas davantage la photographie à une norme esthétique. À suivre le texte du protocole, la réalisation de l'œuvre dure un peu plus de trois minutes. Tous les choix de réalisation de l'œuvre sont effectués dans le but de clarifier la présentation de l'idée qui la sous-tend. Les photographies, enregistrées suivant un dispositif chronologique précis, sont mélangées après tirage. Leur place dans l'ordre chronologique que réclamait le protocole est perdue. L'artiste déclare à ce sujet :

J'ai toujours mélangé l'ordre des photos dans mes accrochages de telle manière que le facteur " temps " ne puisse être perçu comme une séquence d'évènements, mais plutôt comme un seul champ ininterrompu, qui transforme chaque détail en un tout. ${ }^{10}$

La perturbation de l'ordre des photographies dans la séquence participe du même principe que la négligence adoptée quant au traitement photographique du sujet. En effet, les deux démarches sont mises en place pour minimiser l'importance de ce que les photographies représentent sans pour autant

8 Danièle Méaux, "Introduction ", in Protocole et photographie contemporaine, Saint-Étienne, Publications de l'Université de Saint-Étienne, 2013, p. 9.

9 Ibid., p. 9.

10 Propos issus de l'interview du 11-14 octobre 1992 entre Frédéric Paul et Douglas Huebler reproduits dans Frédéric Paul, Douglas Huebler, "Variable », etc., FRAC en Limousin, 1992, p. 118. 
abolir la représentation figurative. Ce dispositif recèle en apparence le paradoxe suivant : révéler un mécanisme implique d'en perturber le résultat visible. L'étude de la perturbation des résultats visibles d'un mécanisme dans le but de comprendre ce dernier n'est pas neuve en soi. Certaines recherches, dans d'autres domaines, se sont appuyées sur une telle méthodologie. Cependant la prise en charge de cette perturbation par Huebler exprime les intentions didactiques de ce dernier à l'adresse des spectateurs.

Les photographies de l'artiste doivent être considérées comme sans-art, comme " anesthétiques " ${ }^{11}$ au regard des critères de son époque (et encore de la nôtre dans une certaine mesure). Le terme " anesthétique ", tel qu'il a été forgé par Marcel Duchamp, est utilisé délibérément et avec les distinctions qu'il y a lieu d'observer par rapport à une anti-esthétique (qui ne serait en fin de compte qu'une esthétique d'opposition). L'absence même du caractère artistique des photographies que propose Huebler permet d'engager le processus de réflexion qui conduit à l'œuvre d'art.

Observons à présent comment l'artiste limite le potentiel narratif des photographies.

L'erreur photographique qui se manifeste par le flou à travers toute la série de photographies a donc dans cette œuvre une fonction précise. Elle est utilisée, au même titre que la perturbation de l'ordre chronologique des images, pour desservir la photographie en limitant son potentiel narratif. Cette dévalorisation de la photographie a pour effet de focaliser l'attention du spectateur sur le protocole lui-même, d'en favoriser la lecture et de soutenir ainsi la lisibilité du concept. Cette propriété accordée à l'erreur photographique dans les œuvres de Douglas Huebler n'est pas sans rappeler les " performatifs " tels qu'Austin les envisage en 1955 dans la première conférence reprise dans son ouvrage Quand dire, c'est faire ${ }^{12}$. Les performatifs, ou énonciations performatives, à la différence des déclaratives visent à "faire quelque chose ». Elles exécutent une action dans le moment même où elles sont prononcées. Ainsi en va-t-il de l'expression "Oui, je le veux » qui consacre l'union maritale entre deux individus. De telles assertions ne décrivent ni ne constatent quoi que ce soit, elles ne sont ni vraies ni fausses. Il faut cependant remarquer que ce que l'on vise à "faire " en employant l'énonciation performative, exige souvent le concours d'autres éléments que les paroles elles-mêmes ${ }^{13}$. Pour être efficiente, l'énonciation performative requiert un contexte d'énonciation. Dans le cas du "Oui, je le veux », le contexte d'énonciation nécessaire à l'efficience de cette déclaration est bien entendu la cérémonie de mariage.

11 Sur base des propos de Duchamp tenus durant le symposium accompagnant l'exposition «Art of Assemblage » MOMA, New-York, 1961.

12 John Langshaw Austin, Quand dire, c'est faire, Paris, Éditions du Seuil, 1970, p. 37-45.

13 Ibid., p. 25. 
Nous souhaitons souligner l'analogie qui se dessine entre l'énonciation performative et l'erreur photographique chez Huebler. L'erreur photographique, dans son ouvre, a pour objectif de déclencher un mécanisme de réflexion chez le spectateur. Son contexte d'énonciation, nécessaire à l'accomplissement du rôle de l'erreur et à son interprétation est constitué par les photographies combinées au protocole dactylographié. Envisagée de la sorte, l'erreur peut être qualifiée de performative. Elle présente en effet une logique d'action inhérente qui incite à passer de l'analyse de la forme (par exemple, du flou) à l'analyse de la structure (" pourquoi ce flou ?»). L'erreur présente ainsi une dynamique qui permet d'" embrayer " vers un processus critique qui mène au cœur de l'œuvre.

L'intérêt des photographies de Douglas Huebler ne réside pas dans leurs qualités propres. Les photographies doivent être comprises en lien avec le texte dactylographié qui les accompagne. L'objectivité chez Huebler passe par une photographie "pauvre $»^{14}$, ou plus précisément déficiente, pour renvoyer au concept qui sous-tend l'œuvre. Il serait abusif d'y voir une démarche documentaire dans son acception courante. L'artiste déclare : "Je n'attache pas d'importance à la précision ou à l'exhaustivité de la documentation. La documentation ne prouve rien. ${ }^{15}$ L'objectivité, ou la réalité que l'on peut déceler chez Huebler est là où se situe l'art, c'est-à-dire premièrement dans la relation entre l'évènement et sa documentation et deuxièmement dans l'expérience que le spectateur peut avoir de l'œuvre, et ceci quelle qu'en soit sa forme. Ce qui importe pour l'artiste, c'est la perception de l'œuvre par le spectateur. L'œuvre ne peut se compléter que dans son appréhension par ce dernier. L'œuvre d'art ne réside pas à proprement parler dans sa réalisation formelle, mais bien dans les mécanismes intellectuels qu'elle enclenche. Douglas Huebler ne pense pas que " les choses puissent être de l'art. Pour lui, les choses sont simplement des choses. ${ }^{16}$ Il privilégie donc logiquement la démarche sur le résultat. Pour le formuler d'une façon encore différente, ce qui intéresse Huebler, « ce n'est pas de pointer l'énonciation de la signification, c'est de pointer la façon dont se forme la signification. ${ }^{17}$ Les recherches d'Huebler à ce sujet sont en phase

14 Une photographie dénuée d'intention esthétique formelle. Pour Douglas Huebler, les photographies sont des documents visuels réalisés, selon ses termes, "de façon purement mécanique, sans aucune intervention d'ordre esthétique ". Propos issus du cat. Artist and Photographs, Multiple Inc., New York, 1969. Cité dans Frédéric Paul, Douglas Huebler, "Variable ", etc., FRAC en Limousin, 1992, p. 170.

15 Arthur Rose (Joseph Kosuth), Four Interviews, Arts, Février, 1969.

16 "I don't think things are art at all. They are only things. " Propos issus d'une interview du 25 juillet 1969 entre Patricia Norvell et Douglas Huebler reproduits dans Alexander Alberro et Patricia Norvell, op. cit., p. 141-142.

17 Catalogue d'exposition Crocodile Tears, Los Angeles, MOCA, 1986, cité par Frédéric Paul, in Frédéric Paul, op. cit., p. 20. 
avec les écrits d'Umberto Eco sur L'œuvre ouverte ${ }^{18}$. Pour transformer le hasard de la réception de l'œuvre en nœud de possibilités interprétatives, il est nécessaire d'introduire dans l'œuvre un schéma d'organisation ${ }^{19}$.

Synthétisons le mécanisme de l'œuvre développé par l'artiste.

Douglas Huebler construit donc son ouvre autour d'un protocole. Ce protocole est au départ de la production de photographies. Les effets secondaires liés à la réalisation du protocole ${ }^{20}$ se manifestent dans les photographies sous forme d'erreurs (en l'occurrence, de flous). Ces erreurs se présentent comme autant de déictiques que le spectateur aura à décoder. Elles pointent vers la structure de l'œuvre, là où se logent réellement l'art et l'intention de Douglas Huebler. Grâce à l'erreur, il réduit le caractère documentaire et narratif de la photographie en vue d'un embrayage critique vers le cour de l'œuvre, vers la formation de la signification. Pour paraphraser Michel Butor qui s'exprimait à la même époque sur le Nouveau Roman, Huebler engage le spectateur à « la recherche d'une réalité, [...], dont le sens profond est dans le mouvement même de la conscience appréhendant le monde extérieur et dans les rapports qu'elle établit entre les divers éléments de ce monde. ${ }^{21}$

\section{Wrong comme (re-) présentation de l'erreur}

Il en va différemment chez John Baldessari. Chez cet artiste, le protocole n'est pas explicite. Cependant, les titres des œuvres guident amplement le spectateur dans la lecture de la démarche artistique.

Wrong appartient à la série des photo \& text paintings. Cette série initiée entre 1966 et 1968 est composée de toiles sur lesquelles sont transférées des photographies noir et blanc au moyen d'une émulsion photosensible liquide. L'image photographique est complétée par un texte rédigé par Baldessari et réalisé par un peintre de lettrage professionnel engagé par l'artiste.

18 Umberto Eco, L'œuvre ouverte, Paris, Éditions du Seuil, 1962.

19 Ibid., p. 160.

20 John Elster, Le laboureur et ses enfants. Deux essais sur les limites de la rationalité, Paris, Minuit, 1987, p. 33, cité dans Danièle Méaux, op. cit., p. 10.

21 Françoise Baqué, Le Nouveau Roman, Paris, Bordas, 1972, p. 60. 


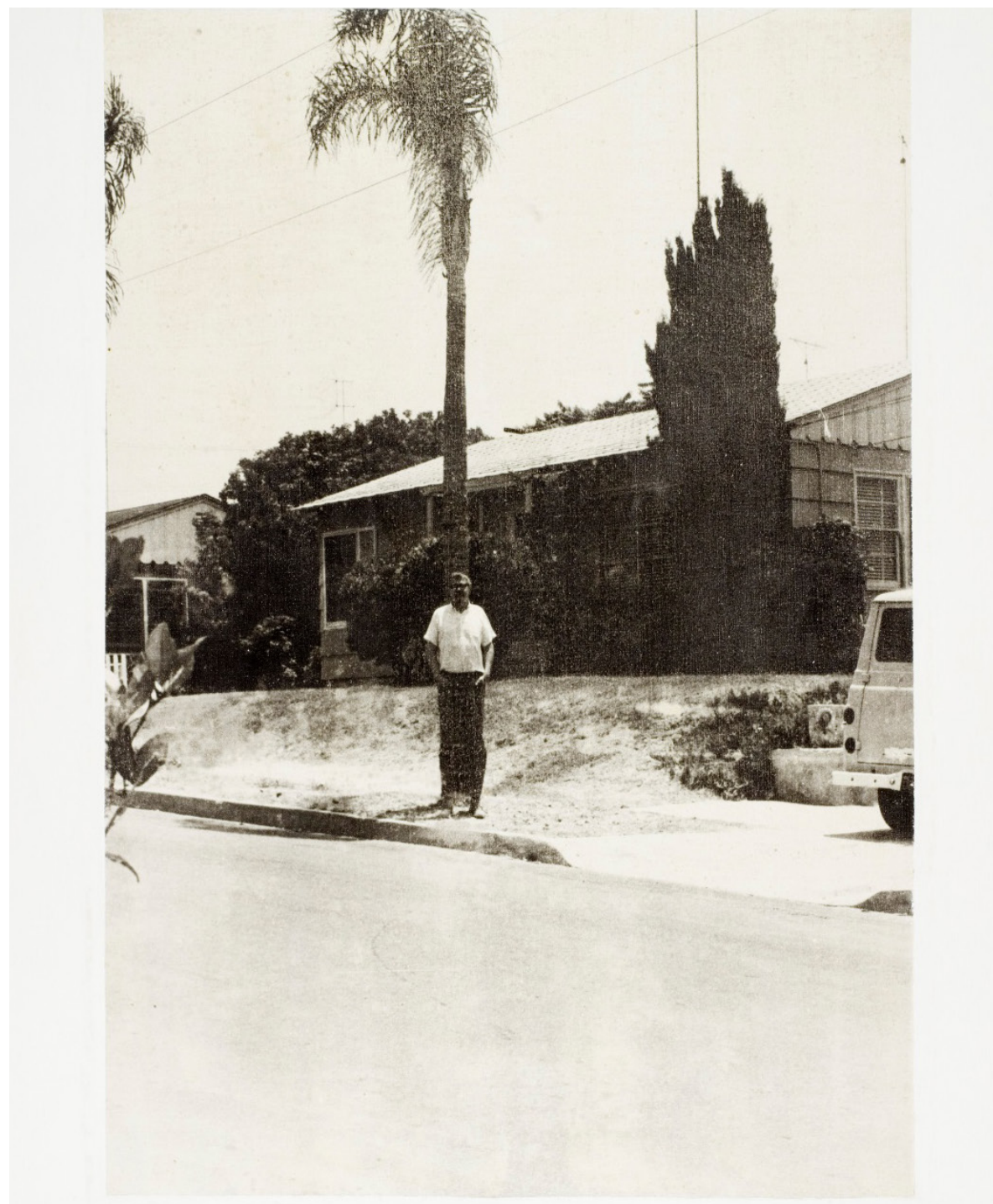

\section{WRONG}

Baldessari, John (b. 1931): Wrong, 1966-68. Contemporary Art Council (M.71.40). Los Angeles (CA), Los Angeles County Museum of Art (LACMA). Photoemulsion with acrylic on canvas, 59 x 45 in (149,86 x 114,3 cm). (C) 2015. Digital Image Museum Associates/LACMA/Art Resource NY/Scala, Florence, Courtesy John Baldessari. 
Wrong, c'est d'abord la transgression d'un conseil de composition. Interrogé sur ses photo \& text, Baldessari parlait de Wrong en ces termes :

J'étais debout devant un palmier - Vous n'êtes pas supposé vous tenir devant un arbre parce que celui-ci semble vous pousser hors de la tête - Cela venait d'une indication issue d'un livre de composition sur la bonne et la mauvaise façon de faire. Il vous donnait la bonne et la mauvaise image. J'adore faire les choses de travers, c'est pourquoi je l'ai appelée Wrong. ${ }^{22}$

Nous aborderons l'analyse de Wrong par le rappel de la notion de lapsus (non-) révélateur. Il arrive que les artistes soient amenés à l'utiliser « intentionnellement ${ }^{23}$. C'est le cas de Baldessari dans Wrong. Dans son argumentation sur le lapsus, Freud traite du poète et du dramaturge. Il ne faut cependant pas forcer le sens du texte pour voir à travers le poète, les traits de l'artiste conceptuel. Le pionnier de la psychanalyse écrivait :

Personne ne songerait à admettre que le poète se soit trompé en écrivant et qu'il ait laissé subsister son erreur, laquelle serait devenue de ce fait un lapsus dans la bouche du personnage. [...] le poète se sert du lapsus comme d'un mot ayant un sens $[\ldots] .^{24}$

Freud fait mention de deux exemples dans la littérature. Il trouve le premier dans Wallenstein ${ }^{25}$ (deuxième partie: Les Piccolomini, premier acte, cinquième scène) et le second (mis en évidence par Otto Rank ${ }^{26}$ ) dans le Marchand de Venise (1596-1597) de Shakespeare (troisième acte, scène deux). Dans les deux cas, les acteurs sont amenés à faire volontairement un lapsus qui va conférer une grande subtilité aux pièces. Suite à ces erreurs, les protagonistes de la pièce et les spectateurs comprennent des éléments encore insoupçonnés. Il ne s'agit donc, bien sûr, pas d'une erreur de l'auteur, mais de la présentation d'une erreur dans le but de donner du sens, voire même, la clé de l'œuvre. Tout comme le poète, Baldessari ne s'est pas trompé. Le lapsus photographique exposé est, nous le savons, calculé pour être présenté. Nous pouvons dire pour paraphraser Freud que l'artiste californien se sert de cette erreur comme d'une image ayant un sens. La force du travail de Baldessari réside dans le fait que l'objet même du lapsus photographique volontaire qu'il met en place est précisément l'erreur. Les lapsus chez les dramaturges évoqués révélaient quelque chose de l'action, celui de Baldessari révèle quelque chose sur le raté et sur l'art. C'est une sorte d'erreur que l'on pourrait qualifier d'auto-référentielle. Baldessari fournit la clé2 ${ }^{27} \mathrm{de}$ son œuvre, sur l'œuvre elle-même en inscrivant wrong en toutes lettres.

22 Moira Roth, A conversation with John Baldessari, University of California, Santa Cruz, 6 janvier 1973 (inédit).

23 Sigmund Freud, Introduction à la psychanalyse (1916), Paris, Éditions Payot, 1985, p. 25.

24 Ibid, p. 25.

25 Trilogie de théâtre de Friedrich Von Schiller créée en 1799.

26 Psychologue et psychanalyste autrichien né en 1884 et mort en 1939.

27 La clé participe également au caractère erroné de l'œuvre. 
Baldessari sélectionne un conseil relatif à la production d'une bonne photographie et s'applique à le transgresser. Deux volontés sont à l'œuvre derrière cette action. La première est une transgression et la seconde est une expérimentation. Sous couvert de transgression et d'expérimentation, Baldessari mène dans Wrong une investigation sur le modernisme ${ }^{28}$. Cette démarche peut être décomposée en plusieurs thèmes distincts. Nous aborderons ici la question de l'appropriation des stratégies des amateurs dans la mesure où elle détermine la réception conceptuelle de la théorie moderniste.

Dans sa quête de la modification de l'image de l'art et de l'artiste, Baldessari utilise régulièrement l'amateurisme. L'appropriation des stratégies des amateurs permet d'opérer une désacralisation de l'Art. Baldessari reproduit les travers d'une photographie d'amateur. Il s'agit d'une démarche intentionnelle. Bien qu'il intègre depuis peu de temps la photographie au sein de ses œuvres, sa pratique a commencé durant son adolescence. De plus, sa formation picturale pouvait lui éviter de réaliser les erreurs compositionnelles qu'il commet. Cet amateurisme apparent est donc délibéré. Celui-ci se reconnait notamment à la centration et à la frontalité du sujet. Dans le chef d'un amateur, ce qui nous apparait comme un barbarisme, pour reprendre le terme de Bourdieu, est en réalité l'accomplissement parfait d'une intention ${ }^{29}$, celle de mettre particulièrement en valeur le sujet fixé. Wrong ne cadre pas avec la définition de la photographie moderniste que donne Greenberg. Dans "Four Photographers »", il définit le caractère littéraire de la photographie. Son approche du médium photographique diffère de celle du médium pictural. Pour Clement Greenberg, la spécificité de la photographie ne réside pas dans des critères formels mais bien dans sa capacité à raconter une histoire. Autrement dit, la valeur artistique de la photographie réside dans son caractère anecdotique. La photographie de Baldessari n'est pas conçue pour raconter une histoire. De plus la fonction de la photographie de Wrong semble être testimoniale, c'est-à-dire assumer le rôle d'un témoin non engagé d'un évènement, attestant du degré minimum d'intentionnalité. La photographie confirme la présence d'un homme à un endroit en un moment donné. Au-delà du simple témoignage factuel, la photographie atteste de la relation entre l'individu et le bâti à l'arrière-plan mais elle ne constitue pas pour autant une anecdote. La juxtaposition des deux éléments est également un

28 Le modernisme photographique ne constitue pas une notion théorique homogène. Résumer le modernisme sans être caricatural constitue une gageure. Cependant pour éclairer le lecteur, nous pouvons rappeler que le concept est le fait de plusieurs théoriciens parmi lesquels nous retenons ici Clement Greenberg et John Szarkowski.

29 Pierre Bourdieu, Un art Moyen, Paris, Les éditions de Minuit, p. 60.

30 Clement Greenberg, "Four Photographers " in The New York Review of books, 23 janvier 1964, vol. 1, n 11. Cité dans O'Brian J. Clement Greenberg, The Collected Essays and Criticism, Modernism with a Vengeance, 1957-1969, Chicago, The University of Chicago Press, 1993, p. 183-187. 
phénomène fréquent dans la photographie d'amateur quand elle veut témoigner d'un rapport de propriété. Les deux éléments se solennisent l'un l'autre. Baldessari est le propriétaire de cette maison et la maison est présentée comme la propriété de Baldessari. La photographie a donc pour objectif de témoigner et de sceller une relation. L'aspect esthétique est complètement étranger aux préoccupations de l'artiste lors de la réalisation de cette photographie.

D'autres indices de l'amateurisme déployé sont perceptibles dans les faiblesses de composition et d'exposition de l'image. Outre l'arbre qui semble sortir de la tête de Baldessari, on peut noter un cadrage trop large, inapte à mettre en valeur le sujet, l'absence d'un premier plan intéressant et finalement la mauvaise exposition du sujet principal dont on ne distingue qu'à peine le visage. Le traitement des photographies, chez Baldessari, est "négligé », ce qui ne cadre pas avec la conception d'un théoricien comme John Szarkowski. Ce dernier, successeur d'Edward Steichen au poste de conservateur pour la photographie au Museum of Modern Art de New York, va rechercher les spécificités formelles de la photographie, ses qualités propres. Dans " The Photographer's Eye $\aleph^{31}$, il dégagera des critères tels que la chose elle-même, le détail ou encore le cadrage. Ces critères permettent de constituer une définition formaliste de la photographie. Baldessari ne recherche pas les spécificités formelles de la photographie, ses qualités propres.

Envisageons la portée de la transgression opérée par l'artiste.

La démarche est critique et la critique a une portée pédagogique. Baldessari conteste une façon éculée de transmettre l'art. Il tend vers un changement de paradigme dans la pédagogie artistique. Il ne veut plus incarner l'image du maittre mais bien celle de l'artiste au travail. Baldessari va privilégier le partage d'expérience et non plus l'unique transmission de savoirs ${ }^{32}$. L'artiste pense devoir se détacher des façons de faire du passé. Dans ses premières années d'enseignement au CalArts, Baldessari dirige un cours intitulé " Post-Studio Art : Problems and Issues ». Le cours s'organise autour d'excursions durant lesquelles les étudiants sont amenés à réaliser des sortes de missions artistiques. Cette démarche annonce certaines entreprises artistiques postmodernes qui caractériseront la Picture Generatio ${ }^{33}$. La contestation est envisagée

31 John Szarkowski, L'œil du photographe, New York, MOMA, 1966.

32 L'accent est mis sur la chose en train de se faire, sur l'expérience et non plus sur le résultat. C'est ce que nous dit Baldessari lorsqu'il reproduit l'expérience de Duchamp avec le mètre-étalon. Lorsqu'en 1913-1914, Marcel Duchamp réalise Trois Stoppages-étalon, ce qui l'intéresse, c'est le résultat de l'objet tombé au sol. Quand en 1975, Baldessari laisse tomber un mètre de nylon dans Strobe Series/ Futurist: Dropping a Cord One Meter Long One Meter, ce qu'il enregistre c'est une démarche. Il évite d'ailleurs de photographier le résultat de l'action.

33 Il s'agit d'une génération d'artistes émergents dans les années soixante-dix. Leur production est fortement marquée par le contexte sociopolitique américain de l'époque. Parmi ces artistes, nous pouvons citer Cindy Sherman, Richard Prince, Barbara Kruger ou encore Louise Lawler. 
comme un moteur d'ouverture. Wrong est un acte dissident, une transgression d'une richesse insoupçonnée. Dans Wrong, en effet, il y a transgression (la transgression d'une consigne de composition), affirmation ou confirmation de cette transgression par l'écrit et validation ou autorisation de la transgression par la présence de l'artiste dans l'œuvre. Le fait que Wrong soit un autoportrait nous indique l'intention de l'artiste d'être associé à l'acte théorique qu'il pose. Cette démarche sera également de mise dans une autre œuvre de la même époque intitulée The Spectator is Compelled to Look Directly Down The Road and Into The Middel of The Picture ${ }^{34}$ de 1966-1968. Dans cette ouvre, Baldessari est debout dans la rue devant chez lui et s'applique cette fois à transgresser un conseil issu d'un livre sur la perspective ${ }^{35}$. Le caractère irrévérencieux de l'œuvre est renforcé par la perturbation des codes de l'autoportrait, le sujet posant de dos. Outre la transgression de la règle de composition, Baldessari fait également fi, consciemment ou non, d'une règle reprise par Walter Benjamin dans sa Petite Histoire de la Photographie ${ }^{36}$. Cette règle est issue d'un ouvrage d'Henry Hunt Snelling sur la pratique photographique :

Ne regardez jamais l'appareil. Les yeux doivent se fixer sur un objet situé un peu au-dessus ou à côté de l'appareil - mais jamais au-dessous ou sur l'instrument, comme dirigé vers lui ; dans le dernier cas, le visage prend une expression fixe, stupide, immobile, avec sourcils froncés et air triste. ${ }^{37}$

L'artiste réalise une erreur et se permet de l'exposer en tant que telle mais plus encore il fait partie de l'erreur. Il l'a intégrée. Nous pouvons dès lors vraisemblablement considérer Wrong comme étant également un commentaire sur le statut de l'artiste et sur son rôle de créateur/auteur. Baldessari tient à rendre compte de son sentiment d'impossibilité d'enseigner l'art de façon traditionnelle et tente de railler la posture du donneur de leçons. C'est également l'attitude qu'il adopte dans Teaching a Plant The Alphabet de 1972 lorsqu'il entreprend d'enseigner l'alphabet à une plante devant une caméra vidéo.

Un hasard programmé va être désormais mis à contribution.

Quelques années plus tard, Baldessari développe une nouvelle série d'œuvres utilisant la photographie. Throwing Three Balls in the Air to Get a Straight Line (Best of Thirty-Six Attempts) ${ }^{38}$ - 1973 - prend appui sur une démarche qui

34 Le spectateur est contraint de regarder directement le bas de la route et le centre de l'image (notre traduction).

35 Ernest Ralph Norling, Perspective Made Easy: A Step-by-Step Methode for Learning the Basis of Drawing, New York, Macmillan, 1939, 1949. Cité dans Coosje Van Bruggen, John Baldessari, New York, Rizzoli, 1990, p. 32.

36 Walter Benjamin, Petite Histoire de la Photographie (1931), Paris, Alia, 2012.

37 Henry Hunt Snelling, The History and Practice of Art of Photography: or, The Production of Pictures Throught the Agency of Light..., ch. IV., New York, Putnam, 1849, p. 41, cite dans Walter Benjamin, Petite Histoire de la Photographie (1931), Paris, Alia, 2012, p. 20.

38 Lancer trois balles en l'air pour obtenir une ligne droite. (Les meilleures des trente-six tentatives) (notre traduction). 
induit une perturbation aléatoire de la composition de l'image photographique dans le but d'empêcher l'atteinte d'un objectif fixé, dans le but de rater d'une certaine manière. Baldessari va mettre en œuvre deux éléments complémentaires importants dans sa production artistique de cette époque. Ces éléments sont le hasard et le choix arbitraire.

Progressivement, le hasard va être érigé comme principe.

Cette œuvre est issue d'une série d'au moins quatre travaux basés sur le même principe. Chaque ouvre de cette série est réalisée avec trois ou quatre balles orange. Le but de l'exercice étant de lancer les balles dans un ciel bleu afin d'obtenir soit une ligne droite soit une forme géométrique régulière (carré ou triangle équilatéral) et d'en prendre la photographie. Le cadrage des photographies inclut parfois la cime d'arbres ou de palmiers. L'œuvre a vraisemblablement été réalisée avec l'aide de Carol Wixom qui était alors encore son épouse. Dans ses Statements, Baldessari déclare que l'œuvre repose avant tout sur le processus de choix des clichés les plus réussis sur l'ensemble de la pellicule utilisée.

Contrairement aux trois autres œuvres de la série, Throwing Three Balls in the Air to Get a Straight Line (Best of Thirty-Six Attempts) ne semble pas destinée à l'exposition en galerie. L'œuvre, composée de douze photographies couleur de 24,1 par 32,4 cm, est tirée à 2000 exemplaires à Milan aux Éditions Giampaolo Prearo/Galleria Toselli. Les photographies sont accompagnées d'une page titre reprenant toutes les informations ainsi que la miniature d'une des photographies. Une page vierge accompagne l'ensemble.

Dans Throwing three balls in the air to get a straight line. (Best of Thirtysix Attempts), l'artiste entreprend la réalisation d'une entreprise apparemment absurde, car inévitablement vouée à l'échec. Cette démarche a été déclinée sous différentes formes. Citons par exemple Throwing Four Balls in the Air to get a Straight Line (Best of 36 Tries) ${ }^{39}$, Throwing Three Balls in the Air to Get an Equilateral Triangle (Best of 36 Tries) ${ }^{40}$ ou encore Throwing Four Balls in the Air to Get a Square (Best of 36 Tries) ${ }^{41}$. Ces œuvres, qui peuvent paraître absurdes, ne sont pas le témoignage d'une quelconque folie de l'artiste mais constituent, une fois de plus, un commentaire sur la démarche artistique. Plusieurs niveaux de lecture peuvent être appliqués à Throwing three balls in the air to get a straight line. (Best of Thirty-six Attempts). C'est peut-être le côté ludique qui est le plus perceptible dans cette œuvre. John Baldessari n'aurait certainement pas récusé la réflexion de Johan Huizinga :

39 Lancer quatre balles en l'air pour obtenir une ligne droite. (Les meilleures des trente-six tentatives) (notre traduction).

40 Lancer trois balles en l'air pour obtenir un triangle équilatéral. (Les meilleures des trente-six tentatives) (notre traduction).

41 Lancer quatre balles en l'air pour obtenir un carré. (Les meilleures des trente-six tentatives) (notre traduction). 
Le jeu n'a pas une tendance à exclure le sérieux mais le sérieux a une tendance à exclure le jeu et à cause de ça le jeu est d'un ordre supérieur au sérieux. " ${ }^{42}$

L'artiste déclarait à son tour :

"En fait, je n'étais pas si fou parce que je m’en foutais. [...] Je m’amusais et, puisque je n'avais pas à plaire à qui que ce soit, je me disais, au fond, pourquoi ne pas faire ces trucs? Ca va m'amuser. Je crois que c'est une grande leçon $-s^{\prime}$ en foutre. ${ }^{43}$

La démarche, qui n'a pour limites que les trente-six poses de la pellicule photographique, s'apparente à celle d'un jeu d'enfant. La recherche semble naïve. L'œuvre, en surface, trahit aussi le goût de l'artiste pour l'expérimentation. Si on avance dans notre réflexion et que l'on met ces photographies en regard des influences de Baldessari et notamment celles de Fluxus, on peut penser que ces œuvres du début des années soixante-dix sont des tentatives de désacralisation de l'art. Abigail Solomon-Godeau voit dans ces démarches " [...] the jouissance of anarchic subversion, the libertarian joy of upsetting rules, hierarchies, conventions " ${ }^{44}$. C'est également l'exposition de "l'instant décisif $»^{45}$ que l'artiste a manqué. Comme c'est souvent le cas chez Baldessari, le titre de l'œuvre, Throwing three balls in the air to get a straight line. (Best of Thirty-six Attempts), en présente la démarche. L'artiste développe une volonté d'arriver à un but, celui énoncé dans le titre. En même temps, il est conscient de l'impossibilité de l'atteindre. Il essaye sans doute avec honnêteté de l'atteindre pendant la réalisation de l'œuvre mais est conscient de l'incapacité mathématique d'y parvenir. Cette phase de l'œuvre peut déjà correspondre à un commentaire sur l'art et sur la création artistique en général et peut-être, au vu de son parcours, sur la peinture en particulier. L'œuvre peut constituer une caricature désenchantée d'une entreprise artistique sans cesse recommencée, tendant vers la perfection, mais ne l'atteignant jamais. La démarche présente dans cette œuvre rappelle également une conception de la création artistique : créer une forme à partir de ce qui n'en a pas.

À travers ces œuvres, l'artiste développe un commentaire sur la démarche de production artistique.

42 Johan Huizinga, Homo Lundens, 1938. Cité dans Danièle Bourcier et Pek Van Andel, La Sérendipité, le hasard heureux, Paris, Hermann Éditeurs, 2011, p. 90.

43 Barbara Isenberg, State of the Arts, New York, Harper Collins, 2000, p. 320-321.

44 "La jouissance d'une subversion anarchique, la joie libertaire de renverser des règles, des hiérarchies, des conventions " (notre traduction). Hugh Davies et Andreas Ales, John Baldessari: National City, San Diego, Museum of Contemporary Art San Diego, 1996, p. 35.

45 C'est un concept cher aux photoreporters. Il s'agit du moment idéal durant lequel tous les éléments contribuant à la réussite d'une photographie seraient condensés. C'est également le titre d'un texte de 1952 dans lequel Henri Cartier Bresson développe sa conception de la photographie. 
Nous considérions l'œuvre comme une entreprise absurde car vouée à l'échec. Baldessari sait que l'échec, le raté sera à la clé de son entreprise. Dans la pensée classique, l'échec n'était que la non-réalisation d'un projet explicite ${ }^{46}$. Aujourd'hui, Baldessari nous montre qu'en échouant dans l'objectif qu'il s'était fixé, il parvient à réussir dans l'échec. L'artiste californien réitère une performance similaire à celle atteinte dans Wrong. Il atteint le raté sans recours à l'accident, mais par le concept même de l'œuvre. Dans Wrong, il s'agissait de prendre le contre-pied d'un précepte de photographie pour amateur. Ici, il s'agit de trouver une action impossible à réaliser et de s'attacher à la faire. Baldessari sait à l'avance qu'il va présenter une erreur, un raté. On peut dès lors considérer que Throwing three balls in the air to get a straight line. (Best of Thirtysix Attempts) est une œuvre réussie. Elle ne réussit pas l'exercice proposé dans son titre. Elle présente l'incapacité d'y parvenir. S’il fallait encore une preuve de cette recherche de présentation de l'incapacité à atteindre le but, il faudrait la voir dans le nombre de photographies qui compose l'œuvre. Douze photographies (les meilleures parmi trente-six) témoignent d'un processus en direction d'un résultat qui lui-même prend son sens de l'échec même à y parvenir.

L'artiste pédagogue nous initie à une production qui réfléchit l'impossibilité de la prétention esthétique, c'est-à-dire, d'une part, la nécessité de cette prétention et, d'autre part, la nécessité d'en prendre distance pour inscrire l'acte créateur dans sa juste dimension, pour le concrétiser.

\section{La construction de l'échec. Photographies ratées, œuvres réussies}

Les trois œuvres envisagées trahissent le regard aiguisé de Douglas Huebler et de John Baldessari sur la démarche de création artistique et sur l'histoire de l'art. Envisager le travail de ces artistes sous l'angle de l'erreur photographique nous a permis de dégager la structure et le fonctionnement du mécanisme de chaque œuvre. Le thème de l'erreur photographique est traité différemment par les deux artistes.

L'observation, la localisation et la définition de l'erreur photographique au sein de la pièce que nous propose Douglas Huebler fait apparaître la stratégie de son œuvre. L'artiste ne rate pas délibérément ses photographies. L'erreur est un effet secondaire du protocole et se manifeste sur les photographies que celui-ci prétend déterminer. Ces erreurs constituent des clés de décryptage de l'œuvre adressées aux spectateurs. Ce dernier est guidé dans son approche et sa lecture de l'œuvre. Huebler met l'erreur au service d'une démarche que nous pouvons qualifier d'épistémologique. Grâce à l'erreur, il réduit le caractère documentaire et narratif de la photographie en vue d'un embrayage critique vers le cœur de l'œuvre, vers la formation de la signification. 
Le travail de John Baldessari offre une approche différente et complémentaire de l'utilisation artistique de l'erreur photographique. Chez ce dernier, l'erreur prend la forme d'une transgression dans Wrong. La transgression d'un conseil de composition engage le spectateur à une réflexion sur la pédagogie artistique qui véhicule des normes esthétiques et réfléchit également la pratique des photographes amateurs. Throwing three balls in the air to get a straight line propose une réflexion sur la démarche de création artistique et ses limites. L'exercice, ludique en apparence, envisagé sous l'angle de l'erreur photographique, nous livre tout son potentiel critique. Les photographies qui composent les deux œuvres de Baldessari ne constituent pas des accidents. Baldessari ne travaille pas la photographie ratée, il ne la récupère pas. Il l'exploite et la met en scène. Ses photographies présentent un défaut d'un point de vue conceptuel et par là participent au succès de l'œuvre qui réside dans l'exposition de ces ratages.

La sélection de trois œuvres ne saurait être représentative de la variété des utilisations de l'erreur photographique dans l'art conceptuel. Cependant, l'analyse des erreurs décelables dans cette sélection d'œuvres suffit à souligner l'intérêt qu'elles représentent. Que ces erreurs soient manifestes ou bien discrètes, d'ordre plastique ou bien conceptuel, après leur intégration dans un processus créatif, elles deviennent à chaque fois riches d'enseignement. Elles nous invitent tantôt à reconsidérer la relation du spectateur à l'œuvre d'art ainsi qu'à la documentation photographique, tantôt à reconsidérer l'acte de création dans un contexte esthétique américain dominé par le modernisme.

François Maheu

Centre de Recherche en Théorie des Arts

Université Catholique de Louvain francois.maheu@uclouvain.be

\section{Résumé}

Notre travail vise à mettre en exergue les bénéfices liés à l'étude de l'erreur photographique dans les démarches de deux artistes conceptuels majeurs. Grâce à l'utilisation d'un protocole engendrant l'erreur photographique, Douglas Huebler enclenche un processus réflexif dans le chef du spectateur. John Baldessari, quant à lui, nous propose deux œuvres, deux représentations de l'erreur photographique mise au service d'une critique de la production artistique et de son enseignement.

\section{Mots-clés}

Art conceptuel, Douglas Huebler, erreur photographique, John Baldessari, stratégie artistique.

\section{Abstract}

Our research aims to highlight the benefits of studying photographic error in the works of two major conceptual artists. Using a procedure that engenders such photographic error, Douglas Huebler catalyzes a process of self-reflection in the spectator. John Baldessari, in turn, proposes two artworks: two representations of photographic error that criticize both the production of art as well as its teaching.

\section{Keywords}

Conceptual Art, Douglas Huebler, photographic error, John Baldessari, artistic strategy. 